\title{
EchoGéo
}

34 | 2015

Varia

\section{Émigration paysanne et vulnérabilité des territoires ruraux dans les Andes équatoriennes}

Une analyse en image depuis la périphérie de Cuenca

\section{Nasser Rebaï}

\section{OpenEdition}

\section{Journals}

Édition électronique

URL : https://journals.openedition.org/echogeo/14420

DOI : $10.4000 /$ echogeo. 14420

ISSN : 1963-1197

Éditeur

Pôle de recherche pour l'organisation et la diffusion de l'information géographique (CNRS UMR 8586)

\section{Référence électronique}

Nasser Rebaï, «Émigration paysanne et vulnérabilité des territoires ruraux dans les Andes

équatoriennes », EchoGéo [En ligne], 34 | 2015, mis en ligne le 15 décembre 2015, consulté le 31 juillet 2021. URL : http://journals.openedition.org/echogeo/14420 ; DOI : https://doi.org/10.4000/echogeo. 14420

Ce document a été généré automatiquement le 31 juillet 2021

EchoGéo est mis à disposition selon les termes de la licence Creative Commons Attribution - Pas d'Utilisation Commerciale - Pas de Modification 4.0 International (CC BY-NC-ND) 


\section{Émigration paysanne et vulnérabilité des territoires ruraux dans les Andes équatoriennes}

Une analyse en image depuis la périphérie de Cuenca

\section{Nasser Rebaï}

"Je ne veux pas que vous galériez comme moi, regardez mes mains, le ciment les a bouffées ». Mohamed Rebaï (1936-2014)

\section{Introduction}

1 Alors que l'émigration internationale des paysans des Andes équatoriennes a été particulièrement importante au cours des deux dernières décennies (Rebaï, 2013), il convient, de s'interroger sur ses conséquences dans les territoires ruraux. Cette réflexion s'avère d'autant plus importante que la rénovation récente du discours politique en Equateur, avec l'avènement de la « Révolution Citoyenne ", menée par le Président Rafael Correa, et l'importance accordée au «Buen Vivir » et à la souveraineté alimentaire, qui occupent une place de choix dans la Constitution de 2008, suggère de s'interroger sur le rôle des paysans dans le processus de construction nationale, alors même que leur émigration témoigne de leur grande vulnérabilité. Le texte qui suit propose donc de s'intéresser à la situation actuelle des campagnes de la sierra équatorienne marquées par une dynamique migratoire importante et de fournir, en outre, des éléments pour penser l'avenir de la paysannerie et le développement des territoires ruraux dans cette région. 


\section{Les mobilités paysannes : un critère d'analyse essentiel pour comprendre la dynamique des territoires ruraux dans les Andes}

2 Ces dernières années, les études sur les mobilités paysannes et leurs effets sur l'organisation des territoires ruraux dans les Andes ont fait l'objet de nombreuses publications. Dans la continuité des travaux de J. Murra (1975) et d'U. Oberem (1981), qui ont montré que la circulation des populations précolombiennes avait pour fonction de tirer profit de milieux écologiques situés à différentes altitudes pour diversifier les productions agricoles, M. Charbonneau (2008) a décrit l'importance ancienne des mobilités paysannes dans la puna péruvienne. Dans cette région l'activité pastorale a historiquement marqué l'organisation du territoire en favorisant la dispersion de l'habitat et les déplacements réguliers des éleveurs sur de longues distances. Cependant le regroupement de la population paysanne qui s'opère progressivement depuis plus d'une vingtaine d'années implique une réorganisation, à l'échelle familiale, de la gestion technique et spatiale du troupeau et pourrait contribuer, dans le futur, à la transformation de l'activité pastorale. De son côté, G. Cortes (2000), à partir de recherches conduites dans la région de Cochabamba, en Bolivie, a rappelé que l'émigration internationale des paysans, plutôt que d'être envisagée comme un exil sans retour, devait être perçue comme une stratégie de maintien de la paysannerie, concrétisée notamment par l'achat de terres.

3 En Équateur, plusieurs études (Chiriboga, 1984; Martínez, 1985; Rebaï, 2009) ont montré que les mobilités de travail, vers les grands centres urbains nationaux ou les grandes zones d'agriculture capitaliste de la côte, constituaient le principal moyen d'intégration économique des populations paysannes de la sierra puisque l'exiguïté de leurs exploitations, qui s'était maintenue en raison d'une réforme agraire aux effets limités (Larrea, 2008), ne leur permettait pas d'entrer dans un processus d'accumulation. En ce qui concerne plus particulièrement la province de l'Azuay, qui constitue notre principale zone d'étude depuis plusieurs années, nos travaux antérieurs (Rebaï, 2012) ont rappelé que la mobilité des paysans y avait fortement évolué au cours des dernières décennies, en raison de crises économiques successives qu'a connu le pays. Dépassant progressivement les cadres provincial et national, la mobilité des individus y est devenue internationale au milieu des années 1960. Cette extension des aires d'émigration a provoqué leur absence prolongée des unités de production. Dès lors, nous nous sommes intéressés à l'évolution de l'agriculture familiale azuayenne et nous avons souligné que les influences croisées de l'émigration paysanne et de la croissance urbaine régionale avaient contribué à l'émergence d'organisations de petits producteurs et à l'essor d'une agriculture marchande dans la périphérie de Cuenca (330 000 habitants), la troisième ville équatorienne. Toutefois, nous avons précisé que cette dynamique commerciale demeurait limitée. Elle ne concernait que 300 petits producteurs à la fin des années 2000 , alors que la province de l'Azuay compte près de 70000 exploitations de moins de 5 hectares (Institut National de Statistiques et de Recensements - INEC, 2002). À l'échelle de la paroisse (village) Octavio Cordero Palacios située à une vingtaine de kilomètres au nord de Cuenca, nous avons mis en évidence les inégalités entre les exploitations. Nous avons montré que les remesas (revenus migratoires) ne servaient à la modernisation des unités de production que si celles-ci disposaient d'un accès direct au marché urbain régional, en intégrant par exemple une 
association de petits producteurs. Or cet accès au marché ne concerne encore aujourd'hui qu'une minorité d'entre elles.

\section{Précisions méthodologiques}

4 L'objectif de ce nouvel article, qui s'inscrit dans la continuité d'un premier texte publié dans la rubrique «Sur l'image » de la revue Echogéo (Rebaï, 2011), est de rendre compte avec davantage de précision de la réalité des territoires ruraux situés dans la périphérie de Cuenca, caractérisés par une forte émigration paysanne et par une intégration marchande limitée des agriculteurs familiaux. Pour ce faire, nous nous appuierons de nouveau sur nos travaux de terrain réalisés entre 2008 et 2010 dans la paroisse Octavio Cordero Palacios, mais également sur les résultats d'une recherche que nous avons menée en 2014 dans la paroisse San José de Raranga, située à une quarantaine de kilomètres au sud de Cuenca (cf. illustration 1). Sur ces deux terrains, nous avons cherché à comprendre comment les logiques productives des exploitations familiales avaient évolué dans le contexte migratoire et quelles en avaient été les conséquences, d'abord paysagères, puis au niveau des économies domestiques.

$5 \quad$ Afin de présenter le plus clairement possible les principaux résultats de ces recherches de terrain, nous utiliserons dans cet article trois photographies à partir desquelles nous inviterons le lecteur (de paysage) à observer certains éléments singuliers. Puis, toujours à partir des clichés proposés, nous décrirons les principales dynamiques productives et les transformations récentes des usages du sol dans les paroisses Octavio Cordero Palacios et San José de Raranga. Pour cela, l'utilisation de données diachroniques aurait été particulièrement utile, mais l'existence de statistiques fiables sur les dynamiques agraires à l'échelle des localités faisant défaut, nous insisterons davantage sur les changements des choix productifs des exploitations en nous référant à nos observations de terrain et aux entretiens que nous avons réalisés avec la population à propos de l'évolution des usages du sol. De même, les Enquêtes Continues sur l'évolution des Superficies et des Productions Agricoles (EPSAC), réalisées par l'INEC et actualisées chaque année, auraient pu nous aider à dresser la tendance de la dynamique agraire à l'échelle de la province de l'Azuay depuis le début des années 2000, mais celles-ci n'étant pas fiables, en raison d'une méthodologie critiquable (Rebaï, 2012), nous assumons le choix de ne pas en faire usage dans cet article, dans lequel nous utiliserons toutefois certaines données générales du dernier Recensement Agraire National (INEC, 2002).

6 Enfin, nous consacrerons la dernière partie de notre texte aux résultats de 10 enquêtes économiques que nous avons réalisées dans la paroisse San José de Raranga et qui nous permettrons d'affiner notre réflexion sur la situation actuelle de la paysannerie dans la région cuencanaise. Ce travail d'enquête a été conduit en parallèle d'une autre recherche dans la province du Cotopaxi ${ }^{1}$, c'est la raison pour laquelle notre échantillon d'étude peut apparaître limité. Toutefois, le but de notre travail dans la paroisse San José de Raranga n'était pas de créer une nouvelle base d'informations statistiques. Il s'agissait plutôt de compléter les résultats des enquêtes économiques que nous avons publiées sur la paroisse Octavio Cordero Palacios (Rebaï, 2011, 2013 et 2014). Ces deux enquêtes, réalisées à seulement quatre années d'intervalle dans la même région, montrent la validité de notre analyse des dynamiques territoriales dans la périphérie rurale de Cuenca. 
Illustration 1 - Carte de localisation des paroisses Octavio Cordero Palacios et San José de Raranga

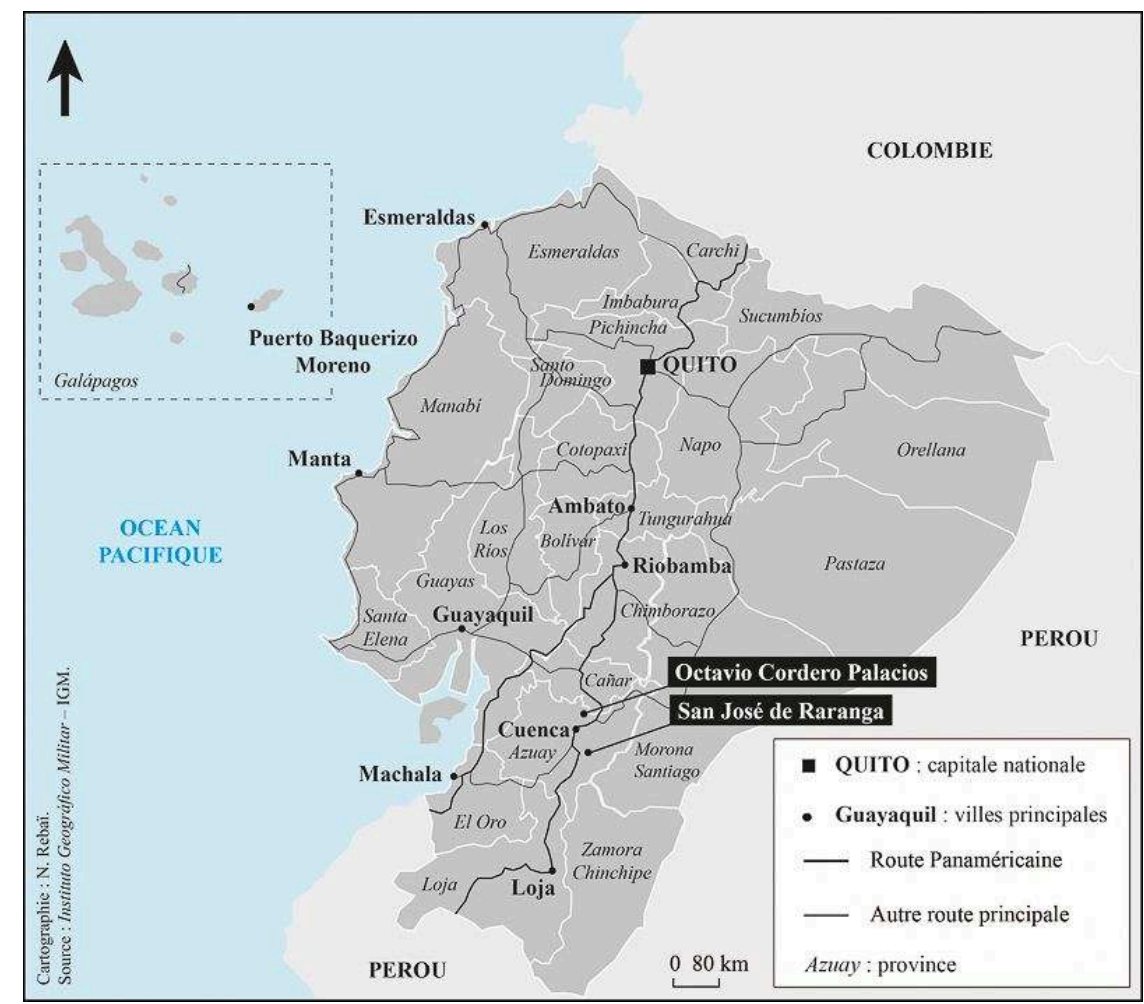

Source : Instituto Geográfico Militar - IGM ; cartographie : N. Rebaï.

\section{Développement de l'élevage laitier au détriment de l'agriculture vivrière}

7 Ces dernières décennies, plusieurs études ont rappelé que la culture du maïs était particulièrement importante dans la province de l'Azuay. La très grande majorité des familles paysannes se consacrait à la production de cette céréale, base de leur alimentation (Gondard, 1976; Borreoro, 1989 ; Huttel et al., 1999, Rebaï, 2012). Le dernier Recensement Agraire National (INEC, 2002) précise d'ailleurs que $71 \%$ des cent mille exploitations de l'Azuay cultivent du maïs au début du siècle, le plus souvent en association avec du haricot, sur un total de près de quarante mille hectares équivalant à $75,5 \%$ des surfaces dédiées aux cultures non permanentes à l'échelle de la province. Pourtant, sur la photographie ci-dessous (illustration 2), qui offre un aperçu du paysage agraire dans la paroisse San José de Raranga en 2014, la présence de maïs est très réduite. En effet, seules trois ou quatre parcelles de quelques dizaines de mètres carrés chacune sont visibles et ce quelques jours à peine avant le début des récoltes qui ont habituellement lieu au mois d'août. Situées à proximité des maisons, ces petites parcelles apparaissent comme autant de petites tâches blondes éparses dans un ensemble dominé par les teintes vertes des bois, plus foncés, et des pâturages, plus clairs. Au premier plan, les habitations en torchis, plutôt petites, ont conservé leurs toits de tuiles tandis que derrière elles, deux autres maisons de deux étages apparaissent et symbolisent le renouvellement récent de l'habitat dans la zone, avec 
leurs toits de zinc et leurs couleurs plus vives. Ces maisons modernisées témoignent de la dynamique migratoire au sein du village. Comme dans d'autres régions de la sierra équatorienne (Rebaï, 2009 et 2011) les premiers investissements des migrants servent bien souvent à la construction de nouvelles maisons, et permettent ainsi d'entretenir un lien avec le lieu d'origine, tout en le transformant. Toutefois, en dépit de ces constructions, anciennes ou modernes, et malgré la présence, au centre de l'image, du linge en train de sécher, pas une seule personne n'apparaît dans le paysage, aucune fumée ne sort du toit des maisons et pas un attelage, pas une voiture ne sillonne la petite route visible au second plan. Sur ce cliché, la solitude du mouton situé au premier plan est flagrante et la campagne semble presque abandonnée.

Illustration 2 - Le paysage agraire de la paroisse San José de Raranga en 2014

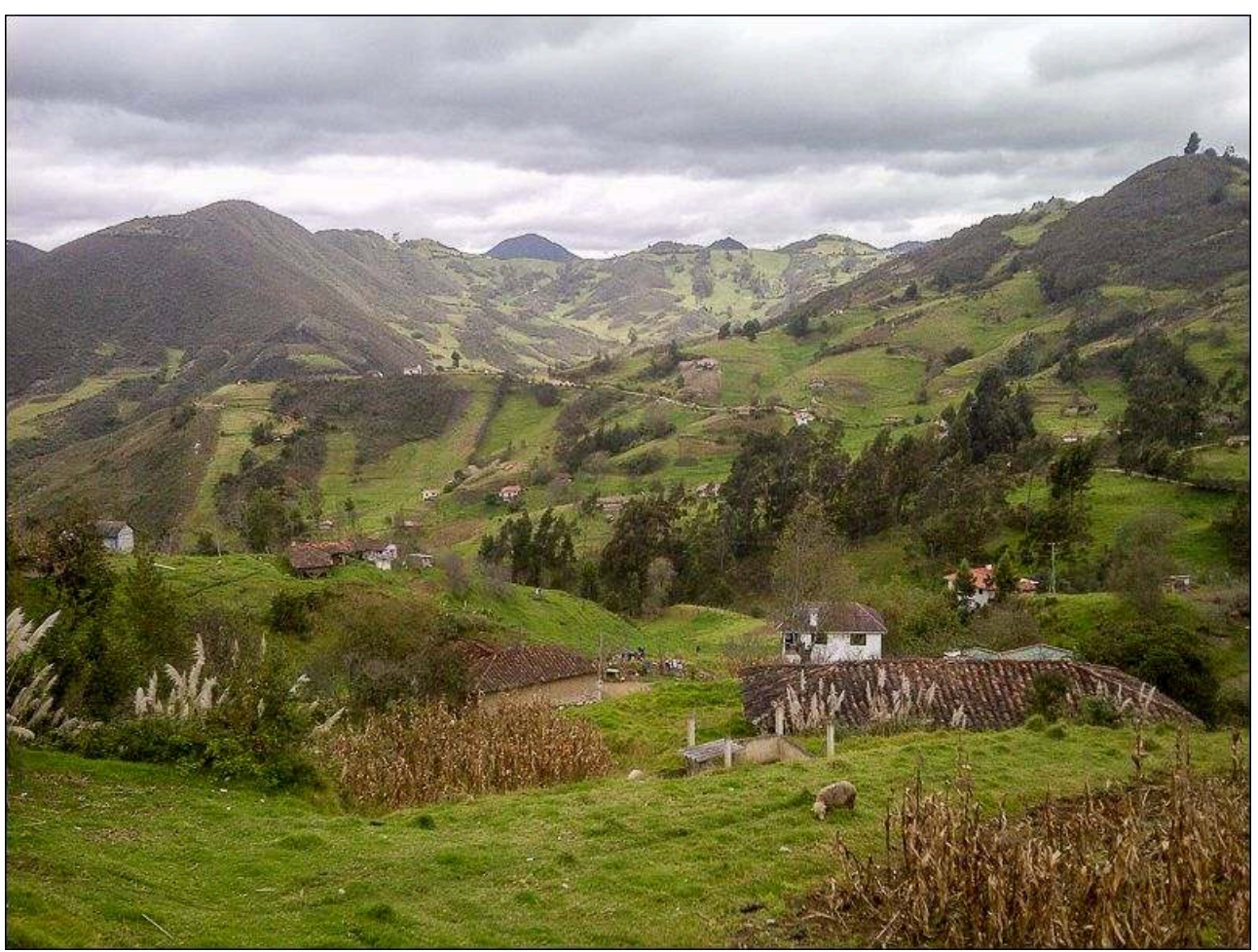

Source : N. Rebaï, 2014.

8 Cette impression se confirme dans les résultats des enquêtes que nous avons réalisées dans la paroisse San José de Raranga. Ils montrent qu'au niveau des dix familles qui composent notre échantillon d'analyse, 21 personnes sur 45 , soit $47 \%$ de la population étudiée, se trouvaient en 2014, et parfois depuis quinze ans, aux États-Unis ${ }^{2}$. Après avoir entendu à plusieurs reprises les personnes nous dire qu'il n'y a "plus personne pour travailler ", nous pouvons penser que la diminution de la main-d'œuvre disponible a contribué à l'abandon progressif des cultures vivrières (maïs, fève, haricot, tubercules). En effet ces cultures sont exigeantes en travail et grosses consommatrices de maind'œuvre durant les périodes de semis, de récolte et de désherbage des parcelles. Elles sont aussi caractérisées par des rendements faibles et sont sujettes à des pertes importantes en cas de mauvaises conditions climatiques. Le manque de main d'œuvre a également conduit les familles de San José de Raranga à délaisser peu à peu les systèmes d'entraide caractéristiques des communautés paysannes andines, comme le cambio mano ${ }^{3}$, pour monnayer toujours plus leurs participations aux tâches agricoles, ce 
qui a provoqué l'augmentation des coûts de production. D'après nos enquêtes, le salaire moyen d'un journalier dans la localité est passé de 5 à 12 dollars entre 2000 et 2014, entraînant une dépense moyenne d'un peu plus de 250 dollars pour cultiver $2500 \mathrm{~m}^{2}$ de maïs. Dans ces conditions les familles paysannes de San José de Raranga ont été contraintes de réduire progressivement la taille des parcelles dédiées au maïs et aux autres cultures vivrières. Les personnes interrogées nous ont expliqué souvent, avec fatalisme, que « cela ne sert à rien de cultiver car cela coûte trop cher ».

Ce constat, nous l'avions déjà fait dans la paroisse Octavio Cordero Palacios où l'émigration paysanne, qui a provoqué la baisse de $30 \%$ de la population locale depuis le début des années 1980, a entraîné la diminution des superficies cultivées (Rebaï, 2012). D'ailleurs, sur la photographie ci-dessous (illustration 3), prise dans cette localité, et dont la perspective se limite cette fois-ci à une seule parcelle d'un demihectare environ, le maïs est totalement absent. À côté de la grande maison bleue, on retrouve à la place du maïs quelques arbres fruitiers dont la production couvre une petite partie des besoins alimentaires d'un migrant de retour, avec qui nous avons réalisé plusieurs entretiens en 2009, et de son épouse. Leurs deux fils, qui vivent aux États-Unis depuis plus de quinze ans, ne sont plus là pour participer aux différentes tâches d'une exploitation d'un peu plus de 4 hectares qui, désormais, si l'on excepte les quelques arbres fruitiers, est entièrement dédiée au pâturage. Ainsi, tout près de la maison, quatre vaches laitières paissent et, non loin d'elles, une rangée de pins et d'eucalyptus indique la limite de la parcelle.

Illustration 3 - Une exploitation laitière dans la paroisse Octavio Cordero Palacios en 2009

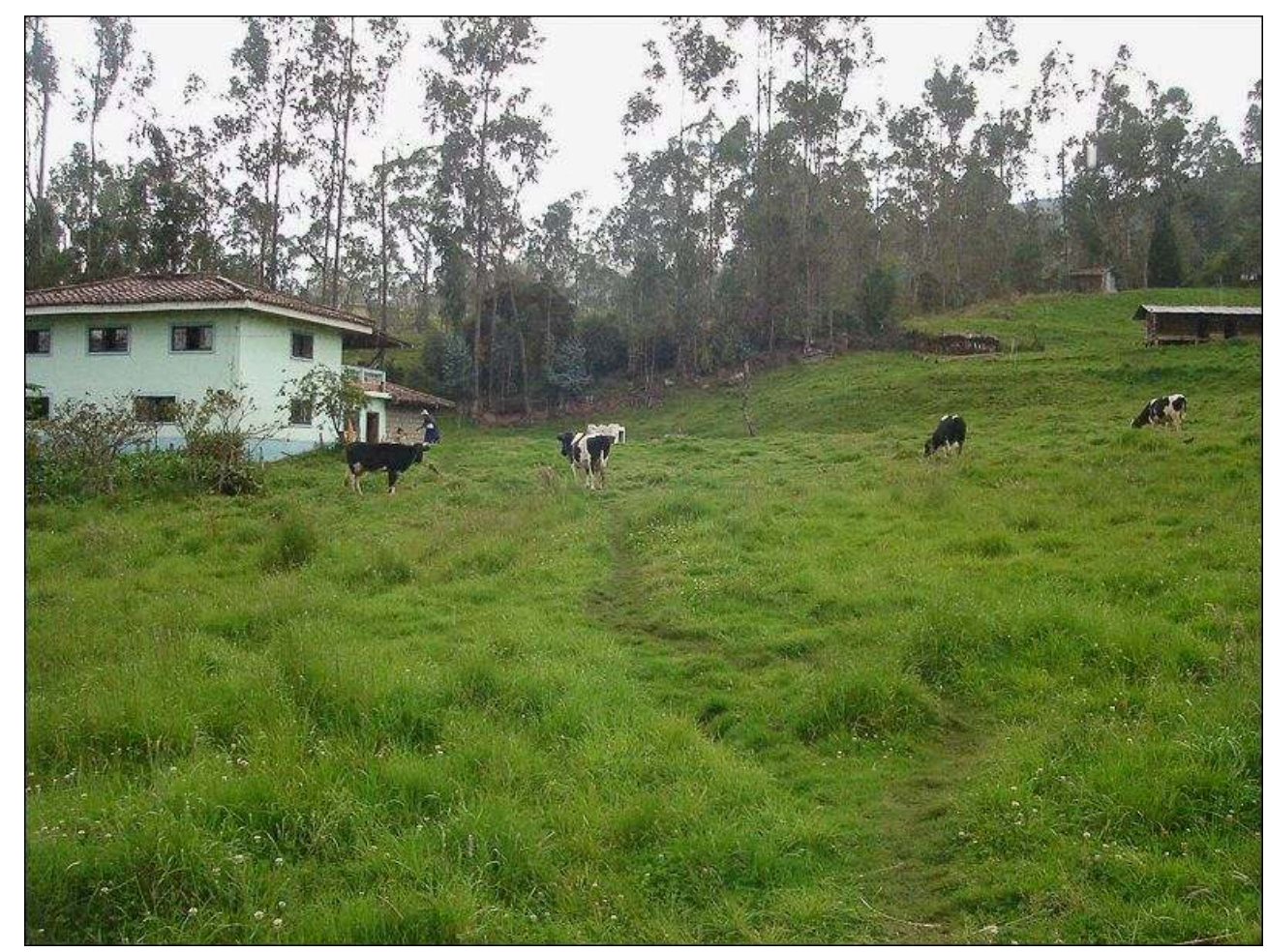

Source : N. Rebaï, 2009.

10 Si la majorité des familles paysannes dans nos deux localités d'étude ont peu à peu délaissé le maïs, devenu trop cher à produire, à l'inverse, comme nous le voyons ici, elles ont privilégié le développement de l'élevage laitier, contraignant il est vrai, mais 
n'impliquant pas de pic de travail obligeant à la mobilisation d'une main-d'œuvre importante à un moment de l'année. L'élevage permet aussi d'obtenir des revenus certes modestes mais réguliers. Ainsi, à Octavio Cordero Palacios, nos enquêtes ont montré que la vente de lait assurait la plus grande partie des revenus agricoles des familles paysannes (Rebaï, 2012). Depuis le début des années 2000, elles ont aussi pu tirer profit d'une politique nationale de protection du marché laitier ayant grandement avantagé les plus petites exploitations de la sierra, leur permettant, dans certains cas, d'entrer dans un processus de capitalisation (Chauveau, 2007 ; Hernández et al., 2013). A San José de Raranga, dans les exploitations que nous avons étudiées en 2014, dont la superficie moyenne est de 2,3 hectares, la charge animale pouvait atteindre 9 têtes de bétail ${ }^{4}$ par hectare. Cette importance de l'élevage s'explique par le fait que, pour les familles, les bovins représentent une forme d'épargne mobilisable dans une situation de crise. Cette surcharge animale n'est pas sans conséquence au sein des exploitations. Elle limite la production quotidienne de lait qui ne dépasse que rarement les 3,5 litres par vache en moyenne. Elle implique l'achat de fourrage, les familles n'ayant pas accès à des terres communales. Elle suppose donc que les familles reçoivent et utilisent des remesas (revenus migratoires). C'est la preuve supplémentaire du maintien d'un lien étroit entre les migrants, leur localité d'origine et l'activité agricole, en dépit de leur absence prolongée, qui conduit nous l'avons dit, à la réduction des superficies cultivées.

11 Malgré une production laitière limitée, les familles de San José de Raranga ont développé ces dernières années une activité de fabrication artisanale de fromages vendus à des intermédiaires qui viennent acheter la production fromagère locale pour aller la revendre à Loja, dans le sud du pays. Les exploitations reçoivent en moyenne 1,05 dollar par livre ( 450 grammes) et parviennent ainsi à obtenir des ressources économiques relativement stables par le biais de cette unique forme d'intégration marchande. Cependant, n'ayant pour seuls acheteurs que ces intermédiaires qui menacent régulièrement de "se fournir ailleurs ", leur pouvoir de négociation demeure très limité et cela les oblige parfois à vendre leurs productions à des prix plus bas, comme nous l'ont expliqué plusieurs personnes que nous avons interrogées. Ainsi, les paysans de San José de Raranga se trouvent dans une situation de subordination à l'égard des intermédiaires qui n'est pas sans rappeler celle des petits producteurs laitiers de Cayambe ${ }^{5}$ vis-à-vis de sociétés agro-industrielles qui, ces dernières années, ont largement contribué à la mutation des territoires ruraux dans la périphérie de Quito, en établissant des relations contractuelles avec de nombreuses unités de production familiales (Martínez, 2013).

\section{Développement des pâturages au détriment du couvert arboré}

12 Si la commercialisation du lait présente des contraintes, la production laitière ne semble en revanche pas sur le point de s'arrêter. Sur la photographie ci-dessous (illustration³), prise à Octavio Cordero Palacios, on observe un bûcheron. Les dizaines d'arbres coupés qui se trouvent autour de lui, prouvent qu'il a effectué un travail très efficace durant les heures précédant notre arrivée. Alors que dans un passé relativement récent les réserves de bois dans cette localité étaient destinées aux usages domestiques et aux activités artisanales (Rebaï, 2012), le cliché pris en 2009 laisse imaginer que leur exploitation actuelle est destinée au secteur de la construction, 
comme en témoignent les centaines de planches proprement entassées. C'est d'autant plus probable que la ville de Cuenca est relativement proche. Ce défrichement massif laisse place désormais à une petite prairie d'une superficie approximative d'un demihectare qui devrait permettre à son propriétaire d'y faire paître ses bovins.

Illustration 4- La disparition du couvert forestier dans la paroisse Octavio Cordero Palacios à la fin des années 2000

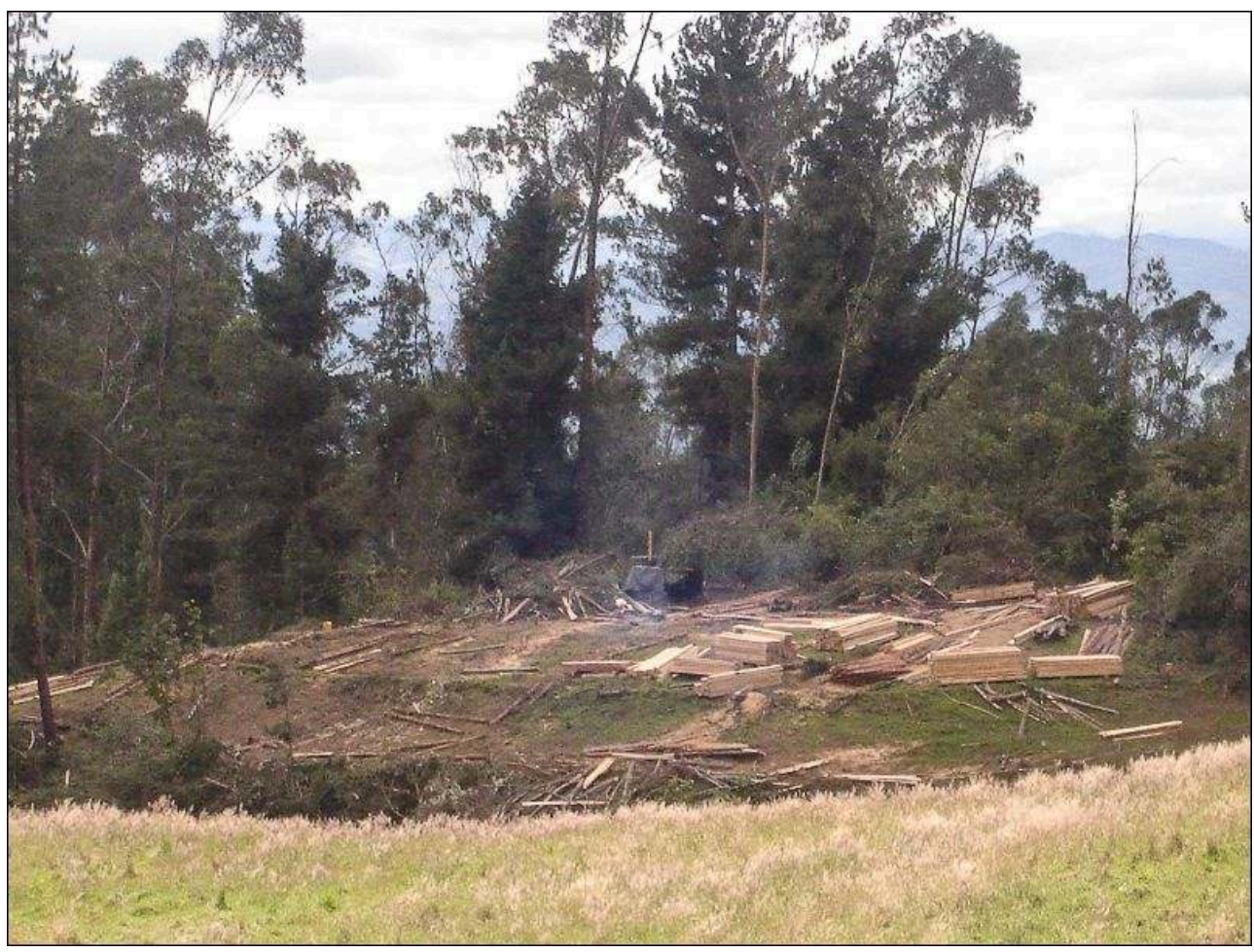

Source : N. Rebaï, 2009.

13 La vente de produits laitiers étant devenue la principale activité économique de nombreuses exploitations d'Octavio Cordero Palacios et de San José de Raranga, beaucoup d'entre elles ont donc fait le choix ces dernières années de faire déboiser leurs parcelles par des bûcherons professionnels. Elles leur ont vendu les arbres qui se trouvaient dans leurs exploitations pour quelques dizaines de dollars, afin de disposer de prairies permanentes pour un plus grand nombre de vaches laitières. C'est pourquoi, désormais, il est fort probable que les pâturages occupent la plus grande partie de l'espace dans nos deux zones d'étude, comme le montre d'ailleurs la photographie (illustration 2) présentée en début de texte. Si l'on se réfère aux seuls résultats de nos enquêtes réalisées dans la paroisse San José de Raranga, à l'échelle des 10 exploitations qui composent notre échantillon d'analyse, et qui représentaient au total 23,3 hectares en $2014,78,8 \%$ de la superficie totale était dédiée aux pâturages, contre seulement $12 \%$ aux espaces boisés et $9,2 \%$ aux cultures.

Dès lors, il convient de souligner un paradoxe. Les informations présentées ci-dessus indiquent que la chute des superficies boisées dans les paroisses Octavio Cordero Palacios et San José de Raranga est liée à la baisse de la population dans ces deux localités, ce qui contredit la forest transition theory (Mather, 1992; Rudel, 1998). Cette théorie veut que les migrations des populations rurales aient pour conséquence de favoriser le retour de la « nature ». En Équateur, ce constat a d'ailleurs été fait, dans les 
provinces du Morona Santiago (Rudel et al., 2002) et de Loja (Gray, 2008), où les baisses de densités rurales ont conduit à la diminution des superficies cultivées et à la formation de forêts secondaires. Cela prouve en définitive qu'il est difficile de théoriser sur les effets de l'émigration en milieu rural compte tenu des contrastes qu'il est possible d'observer d'une région à l'autre en ce qui concerne les usages du sol, lesquels n'évoluent pas seulement en fonction des densités de population. Cela se confirme à l'échelle de nos localités d'étude où la réduction du couvert arboré a lieu dans un contexte économique qui favorise le développement de l'élevage laitier par les petites exploitations familiales, en dépit d'une baisse de la population paysanne.

\section{Contexte migratoire et vulnérabilité économique des exploitations familiales}

Au-delà des mutations des usages du sol, le contexte migratoire et le développement de l'élevage laitier impliquent une transformation de l'économie paysanne. Nos enquêtes réalisées à San José de Raranga montrent que les ventes de produits laitiers constituaient en 2014 la seconde source de revenus réguliers, après les remesas, pour 9 des 10 familles. Néanmoins, cette situation révèle en réalité la vulnérabilité économique des exploitations familiales qui, amputées d'une partie de leur maind'œuvre, sont contraintes de se "spécialiser " dans la production de lait, alors même qu'elles ne disposent pas de grandes ressources foncières. Dans ces conditions, et en délaissant les cultures vivrières, elles se retrouvent dépendantes de l'achat de vivres pour couvrir leurs besoins alimentaires, souvent importants lorsqu'il s'agit de nourrir plusieurs «bouches ». Six des 10 familles que nous avons étudiées en 2014 comptaient entre 4 et 7 membres quotidiennement présents sur l'exploitation, et notamment des enfants et des personnes âgées.

16 Cette vulnérabilité est d'autant plus grande que nos enquêtes révèlent que, pour 8 familles sur 10, les revenus laitiers étaient en 2014 insuffisants pour couvrir intégralement les dépenses de nourriture qui atteignaient en moyenne 209 dollars par mois et concernaient essentiellement l'achat de riz, de pâtes, de pommes de terre, de quelques légumes et d'un peu de matière grasse (huile, beurre ou saindoux). Cela signifie donc que l'élevage laitier ne pourrait pas assurer la reproduction des familles paysannes de San José de Raranga, du moins de celles qui composent notre échantillon d'étude, si les revenus qu'il leur permet d'obtenir, en moyenne 92,3 dollars bruts par mois en 2014, n'étaient pas complétés par des revenus extra-agricoles. Ainsi, pour 9 des 10 familles de notre échantillon d'analyse, dont les revenus moyens atteignaient 391 dollars bruts mensuels en 2014, $57 \%$ des rentrées monétaires régulières provenaient d'activités extra-agricoles, alors que les revenus liés aux petits élevages (cochons d'Inde, volailles, porcs et ovins) plutôt modestes et irréguliers, ne représentaient en moyenne que 65 dollars bruts par mois, soit $16,7 \%$ des revenus moyens. Pour 6 des 10 familles étudiées, les remesas représentaient même 62 \% de leurs revenus globaux, chiffre révélateur de leur vulnérabilité économique. 


\section{Conclusion : au-delà de l'émigration internationale, repenser les mobilités locales et les relations ville- campagne pour le développement durable des territoires ruraux}

À l'image d'autres territoires ruraux de la région andine, ceux de la périphérie de Cuenca connaissent actuellement de profondes mutations sous l'effet d'importantes mobilités paysannes. À l'échelle locale, les paroisses Octavio Cordero Palacios et San José de Raranga, montrent que la baisse de la main-d'œuvre a entraîné une réduction des superficies cultivées et une perte importante d'agro-biodiversité symbolisée par la raréfaction des parcelles de maïs, élément central de l'agriculture andine et de l'alimentation quotidienne des communautés paysannes. Le passage d'une agriculture de subsistance à une activité d'élevage dominante, bien que rémunératrice, a provoqué l'augmentation de la vulnérabilité des familles paysannes, dépendantes des prix fixés par les intermédiaires et contraintes de s'approvisionner sur le marché pour se nourrir. Ainsi, bien que le riz et les pâtes aient remplacé le maïs, la consommation familiale dépend désormais des ventes de lait et des revenus migratoires.

Dans nos deux zones d'étude, l'usage des revenus migratoires au sein des exploitations témoigne de la permanence d'un lien entre les migrants et leur localité d'origine. L'analyse de l'évolution actuelle de la ruralité andine ne doit donc pas être envisagée seulement à l'échelle locale, mais doit nécessairement intégrer la question de la mobilité des individus. Ainsi, dans notre travail, si nous avons pu remarquer que l'émigration internationale permettait aux familles paysannes de disposer de revenus qui pouvaient en partie être investis dans l'élevage, nous avons également souligné que le manque d'intégration marchande, et donc, le manque de circulation des individus et des productions de la campagne vers la ville, renforçait la dépendance des exploitations à l'égard du lait et des remesas, leurs seules ressources monétaires régulières. Si cette dépendance pose évidemment la question de leur sécurité alimentaire, la réduction rapide des surfaces arborées et les surcharges animales dans ces deux zones d'étude posent quant à elles la question de la durabilité du système agraire. Bien que dans le cadre de cet article nous n'ayons pas abordé la dimension environnementale des effets de l'émigration paysanne, nul doute que cette thématique de recherche devrait faire l'objet d'études spécifiques dans les prochaines années en s'inscrivant dans la suite logique de travaux (López, 2004 ; Alomia, 2005 ; Girard 2005, Pohle et al., 2010) qui se sont intéressés aux relations entre les activités agropastorales et la dégradation des sols de haute montagne dans la sierra équatorienne

Si l'on s'en tient à la seule question alimentaire, il convient d'insister à nouveau sur le fait que le contexte migratoire des dernières années a renforcé la dépendance des familles paysannes aux achats de nourriture. On est donc encore très loin du «Buen Vivir » et de la souveraineté alimentaire présentés comme deux priorités de l'actuel gouvernement qui, pour le moment, n'a mené aucune action de grande envergure pour le développement rural dans la région andine. Si l'émigration paysanne a certes diminué ces dernières années (Herrera et al., 2012), elle ne s'est toutefois pas arrêtée. En outre, plusieurs centaines de milliers d'Équatoriens originaires des campagnes continuent de vivre à l'étranger, conscients qu'un retour dans leur localité d'origine ne serait pas viable, en dépit de tentatives gouvernementales de les attirer et aux effets 
donc très limités (Moncayo et Herrera, 2011; Giunta et Corrado, 2014). On est également très loin de la "reconnaissance de l'agriculture familiale et paysanne » dans la région andine que des auteurs comme Heinisch et al. (2014) prétendent déceler parce que se développent ici et là des circuits courts de commercialisation qui, précisons-le, ne satisfont le plus souvent qu'une clientèle privilégiée (Girard, 2015). Car l'immense majorité des paysans des Andes équatoriennes demeure dans une situation de grande vulnérabilité économique et l'émigration, encore perçue comme un moyen de survie, y contribue largement. À moyen terme, cette vulnérabilité devrait s'accentuer en raison d'une augmentation récente des activités extractives (Bustamante et Lara, 2010; Ospina et Lalander, 2012), autre paradoxe de la révolution du "Buen Vivir», et parce que l'Équateur a signé à la fin de l'année 2014 un Traité de Libre-Échange avec l'Union Européenne qui, on peut le craindre, affaiblira un peu plus l'agriculture familiale de ce pays.

En dépit de ce constat, on pourra toutefois être d'accord avec les auteurs précédemment cités sur le fait qu'il est dorénavant nécessaire de changer le modèle de développement rural dans les Andes en valorisant l'agriculture paysanne dans le cadre de relations ville-campagne redéfinies qui favoriseraient l'accès des petites unités de production au marché, et donc au capital. Cela permettant leur modernisation et leur diversification économique progressives (Cochet et al., 2009 ; Rebaï, 2012). En favorisant la «multifonctionnalité » des exploitations familiales (Losh, 2001), capables de combiner productions agropastorales, transformations des produits et ventes directes, dans le cadre de nouvelles formes de circulations entre milieux rural et urbain, un pays comme l'Équateur qui vit actuellement sa « Révolution Citoyenne », pourrait largement contribuer à la réhabilitation de l'agriculture familiale dans ses politiques de développement. Dans ce pays, qui compte aujourd'hui près de $65 \%$ de population urbaine (plus de 10 millions de personnes) et pas moins de 15 villes de plus de 100 mille habitants, la construction de relations étroites entre villes et campagnes, à partir d'une intégration marchande plus importante des agriculteurs familiaux et d'une valorisation plus évidente des ressources locales, serait une stratégie judicieuse pour contribuer à la création d'emplois dans les campagnes et relever le défi de la "souveraineté alimentaire ", si souvent évoqué dans le discours politique ces dernières années.

\section{BIBLIOGRAPHIE}

Alomía M., 2005. Efectos de la producción agropecuaria en los suelos de los páramos: el caso de Guangaje. Ecuador Debate, nº 65, p. 175-194.

Borrero A. L., 1989. El paisaje rural en el Azuay. Cuenca, BCE, 270 p.

Bustamante T., Rommel L. (coordinateurs), 2010. El Dorado o la Caja de Pandora: matices para pensar la minería en Ecuador. Quito, FLACSO Ecuador, 145 p.

Charbonneau M., 2009. Système de peuplement dispersé et regroupement dans les sociétés pastorales andines. Annales de Géographies, n 670, p. 637-658. 
Chauveau C., 2007. La producción lechera en las economías campesinas de la Sierra: seguridad, dinamismo económico y pluriactividad. In Brassel F. et Hidalgo F., Libre comercio y lácteos. La producción de leche en El ecuador entre el mercado nacional y la globalización. Quito, SIPAE/IRD, p. 43-51.

Chriboga M., 1984. Campesino andino y estrategias de empleo: el caso de Salcedo. In SanchezParga (ed.), Estrategias de supervivencia en la comunidad andina. Quito, CAAP, p. 59-124.

Cochet H., Aubron C., Jobbé-Duval M., 2009. Quelles sont les conditions à réunir pour une intégration marchande porteuse de développement durable pour les paysanneries andines? Les cahiers d'Outre-Mer, ${ }^{\circ}{ }^{\circ} 247$, p. 395-417.

Cortes G., 2000. Partir pour rester : survie et mutation de sociétés paysannes andines (Bolivie). Paris, IRD, $413 \mathrm{p}$.

Girard M., Economía social y comercialización de productos orgánicos en Cusco, Perú. ¿Pasos iniciales en el desarrollo de una estrategia alternativa? Eutopía, p. 91-108

Girard S., 2005, Les páramos, espace stratégique pour la gestion de l'eau dans les Andes septentrionales : le bassin versant dur río Ambato (Equateur). M@ppemonde, nํㅜ 78, vol. 2 [en ligne].

Giunta I., Corrado I., 2014. Which role for family and peasant farming in the Plan Tierras y de Retorno Productivo promoted by the Republic of Ecuador? In Colloque International Agricultures familiales, territoires et perspectives de développement dans les Suds. Tunis, IRMC/PRODIG/CIST/ INRAT.

Gondard P., 1976. Zonas agrícolas de la sierra. Boletín del Instituto Panamericano de Geografia e Historia - Sección nacional del Ecuador, nº 9-10, p. 1-7.

Gray C., 2008. Out-Migration and Rural Livelihoods in the Southern Ecuadorian Andes. Tesis Dissertation. Chapel Hill, University of North California, 201 p.

Huttel C., Zebrowski C., Gondard P., 1999. Paisajes agrarios del Ecuador. Quito, IRD/IFEA/PUCE, $285 \mathrm{p}$.

Heinisch C., Gasselin P., Durand G., 2014. Circuits alimentaires de proximité dans les Andes. Vers une reconnaissance de l'agriculture familiale et paysanne. Economie rurale, n ${ }^{\circ} 343$, p. 71-86.

Hernández M., Mafla H., Proaño V., 2013, Articulación del sector lácteos campesino ecuatoriano al Mercado. In Aubron C., Hernández M., Lacroix P., Mafla H. et Proaño V. (eds.), Producción campesina lechera en los países andinos: dinámicas de articulación a los mercados. Quito, AVSF/SIPAE, p. $129-162$.

Herrera G., Moncayo M. I. et Escobar García A., 2012. Perfil migratorio del Ecuador 2011. Quito, OIM, $128 \mathrm{p}$.

INEC - Instituto Nacional de Estadisticas y Censos, 1982, IV Censo de Población.

INEC - Instituto Nacional de Estadisticas y Censos, 2001, VI Censo de Población.

INEC - Instituto Nacional de Estadisticas y Censos, 2002, III Censo nacional agropecuario.

INEC - Instituto Nacional de Estadisticas y Censos, 2010, VII Censo de Población.

Larrea C., 2008, Tenencia de la tierra, cambios agrarios y etnicidad indigena en el Ecuador: 1954-2000. In North L. et Cameron J., (eds.), Desarrollo rural y neoliberalismo. Ecuador desde una perspectiva comparativa. Quito, UASB, 129-146. 
Losh B., 2003. La multifonctionnalité face aux défis des agricultures des Suds : une perspective de refondation de refondation des politiques publiques. In Barthélémy D., Delorme H., Losch B., Moreddu C. et Nieddu M. (eds.), La multifonctionnalité de l'activité agricole et sa reconnaissance par les politiques publiques. Dijon, Educagri-CIRAD, p. 165-192.

Lopez M.F., 2004. Agricultural and Settlement Frontiers in the Tropical Andes: The Páramo Belt of Nothern Ecuador, 1960-1990. Regensburg, Institut für Geographie an der Universität, 180 p.

Martínez L., 1985. Migración y cambios en las estrategias familiares de las comunidades indígenas de la Sierra. Ecuador Debate, $n^{\circ}$ 8, p. 110-152.

Martínez D., 2013. La asociación lechera, ¿Desarrollo lo cal o subordinación productiva? El caso de la co munidad La Chimba, Cayambe. Ecuador Debate, no 89, p. 119-133.

Mather A., 1992. The forest transition, Area, vol. 24, no 4, p. 367-379.

Moncayo M. I., Herrera G., 2011, El programa « Bienvenid@s a casa ». Estudio sobre la experiencia del fondo « El cucayo ». Avances de Investigación, $\mathrm{n}^{\circ}$ 51. Madrid, CeALCI-Fundación Carolina, 124 p.

Murra J., 1973. Formaciones económicas y políticas del mundo andino. Lima, Instituto de Estudios Peruanos, $339 \mathrm{p}$.

Oberem U., 1981. El acceso a recursos naturales de diferentes ecologías en la sierra ecuatoriana (siglo XVI). In Moreno Yánez S. et Oberem U. (eds.), Contribución a la etnohistoria ecuatoriana. Quito, IOA, p. 45-71.

Ospina P., Lalander R., 2012. Razones de un distanciamiento político: el Movimiento Indígena ecuatoriano y la Revolución Ciudadana, OSAL, n 32, p. 117-134.

Pohle P., Gerique A., Park M., López M.F., 2010. Human ecological dimensions in sustainable utilization and conservation of tropical mountain rain forests under global change in southern Ecuador. In Tscharntke T., Leuschner C., Veldkamp E., Faust H., Guhardja E., Bidin A. (eds.), Tropical Rainforests and Agroforests under Global Change: Ecological and Socio-economics Valuations. Berlin, Springer Science, p. 477-509.

Rebaï N., 2009. De la parcelle à l'archipel: mobilité paysanne et construction territoriale dans les Andes équatoriennes, Revue Interdisciplinaire de Travaux sur les Amériques, $\mathrm{n}^{\circ} 2$ [en ligne].

Rebaï N., 2011. Les effets de l'émigration paysanne dans les Andes équatoriennes : une lecture photographique. EchoGéo [En ligne], 16 | 2011, mis en ligne le 04 juillet 2011. URL : http:// echogeo.revues.org/12382; DOI : 10.4000/echogeo.12382

Rebaï N., 2012, A chacun son chemin. Une analyse de la redéfinition des stratégies paysannes et des dynamiques territoriales dans le contexte migratoire des Andes équatoriennes. Thèse de doctorat. Paris, Université Paris 1, 346 p.

Rebaï N., 2013. Quand l'argent de la migration change la donne : développement agricole et dynamique foncière dans une localité de la province andine de l'Azuay (Equateur). Autrepart, dossier thématique « L'argent des migrations », nº 68, p. 193-212.

Rebaï N., 2014. Rôle des productrices maraîchères dans l'approvisionnement de la ville de Cuenca en Equateur, Pour - dossier thématique « L'agriculture familiale à travers le prisme du genre »$n^{\circ} 222$, p. 261-273.

Rudel T., 1998. Is there a forest transition? Deforestation, reforestation, and development. Rural Sociology, vol. 63, no 4, p. 533-552. 
Rudel T., Bates D., Machinguiashi R., 2002. A tropical forest transition? Agricultural change, Outmigration, and secondary forests in the ecuadorian amazon. Annals of the Association of American Geographers, vol. 92, n 1, p. 87-102.

Sanchez-Parga J., 2014. Alternativas virtuales vs. cambios reales: derechos de la naturaleza, buen vivir, economía solidaria. Quito, CAAP, 127 p.

\section{NOTES}

1. Dans cette province, nous avons mené des enquêtes dans le cadre du projet de recherche «MAN-PEST » qui porte sur la sécurité alimentaire des communautés paysannes andines.

2. Ce premier résultat est particulièrement important parce qu'il contraste très nettement avec les chiffres du dernier recensement démographique qui indique que la population de la paroisse San José de Raranga a augmenté de $15 \%$ entre 2001 et 2010, en passant de 2050 à 2351 habitants (INEC, 2001/2010), alors même que la localité a enregistré une baisse de sa natalité (la classe d'âge des 0/9 ans est passée de 626 à 574 individus entre 2001 et 2010), et peu de retours de migrants (nationaux et internationaux) au cours de cette dizaine d'années. En effet, le dernier recensement rend compte du retour de 22 personnes qui vivaient à l'étranger et de 13 autres personnes qui se trouvaient dans une autre province que celle de l'Azuay mais qui vivaient en Équateur cinq ans auparavant. Ces éléments ne peuvent aller dans le sens d'une augmentation démographique locale de 301 personnes, d'autant plus que la paroisse San José de Raranga est loin d'être une localité économiquement attractive où de nouvelles populations viendraient s'installer ou une zone où les cuencanais choisiraient de s'établir pour bénéficier d'un meilleur cadre de vie. Nous supposons par conséquent qu'au moment de répondre aux enquêteurs de l'INEC, de nombreuses personnes ont déclaré que les membres émigrés de leur famille vivaient dans la localité, craignant comme c'est parfois le cas que les pouvoirs publics puissent leur confisquer leurs terres en cas d'absence de leurs parents ou de de leurs enfants. En résumé, nous émettons l'hypothèse qu'une erreur soit apparue dans le recensement de la population de la paroisse San José de Raranga en 2010.

3. Ou prestamano. Terme espagnol ayant remplacé l'ayni quichua et qui désigne les échanges réciproques de travail entre parents ou membres d'une même communauté paysanne.

4. Dans certains cas, il pouvait s'agir uniquement de gros bétail (taureaux et vaches laitières).

5. Dans la province du Pichincha.

\section{RÉSUMÉS}

Dans les Andes équatoriennes, et plus particulièrement dans la province de l'Azuay, l'émigration paysanne apparait comme un symbole fort de la vulnérabilité des agriculteurs familiaux. Cette émigration, très importante dans certaines zones, implique de profondes transformations des territoires ruraux, en témoigne la réduction drastique des superficies cultivées qui marque l'accentuation de la vulnérabilité des exploitations familiales. Nous abordons les dimensions paysagère et économique des effets de l'émigration paysanne dans la sierra équatorienne à partir de l'étude de deux localités situées dans la périphérie de la ville de Cuenca. Puis nous orientons 
notre propos vers la nécessité de repenser le développement des territoires ruraux andins à partir d'une redéfinition des relations ville-campagne.

In the Ecuadorian Andes, particularly in the province of Azuay, peasant migration appears as a strong symbol of the vulnerability of family farmers. This emigration, in very large numbers in some areas, logically implies major transformations of rural territories evidenced by the drastic reduction of the cultivated areas which marks the increased vulnerability of family smallholdings. In this article, which will focus on two localities in the countryside of the city of Cuenca, we will discuss the landscape and economic effects of peasant migration in the Ecuadorian Sierra, before orienting our analysis to the necessity to rethink the development of the rural territories with a redefinition of urban-rural linkages.

En los Andes ecuatorianos, y en particular en la provincia del Azuay, la migración campesina aparece como un símbolo evidente de la vulnerabilidad de los agricultores familiares. Esta migración, muy importante en algunas zonas, implica profundas transformaciones de los territorios rurales, como lo muestra la reducción de las superficies cultivadas que señala la acentuación de la vulnerabilidad de las explotaciones familiares. A través de este artículo, que se centrará en dos localidades de la periferia de la ciudad de Cuenca, estudiaremos las dimensiones paisajística y económica de los efectos de la migración campesina en la sierra ecuatoriana, antes de orientar muestra análisis hacia la necesidad de repensar el desarrollo de los territorios rurales a partir de una redefinición de las relaciones campo-ciudad.

\section{INDEX}

Mots-clés : Equateur, Andes, territoires ruraux, émigration paysanne, vulnérabilité

Palabras claves : Ecuador, Andes, territorios rurales, migración campesina, vulnerabilidad

Keywords : Ecuador, Andes, rural territories, peasant migration, vulnerability

\section{AUTEUR}

\section{NASSER REBAII}

Nasser Rebaï, nass.reb@hotmail.fr, est Chercheur associé à l'UMR PRODIG. Il a publié récemment :

- Rebaï N., 2014. Émigration paysanne et mutation du patrimoine dans les provinces du Cañar et de l'Azuay. Histoire(s) de l'Amérique latine, vol. 11 [en ligne], 18 p.

- Rebaï N., 2014. Rôle des productrices maraîchères dans l'approvisionnement de la ville de Cuenca en Equateur. Pour, $n^{\circ} 222$, p. 261-273.

- Rebaï N., 2013. Quand l'argent de la migration change la donne : développement agricole et dynamique foncière dans une localité de la province andine de l'Azuay (Equateur).

Autrepart, n 68, p. 193-212. 